\title{
Cryo-Fixed Stained Microtubules Can be Imaged with High Electron Doses for Accessing the Full Resolving Power of an Electron Microscope
}

\author{
Andrea Fera ${ }^{1,2}$ and Louis (Chip) Dye ${ }^{3}$ \\ ${ }^{1}$ Unit for Cytoskeletal Dynamics, Eunice Kennedy Shriver National Institute of Child Health and \\ Human Development, NIH. \\ ${ }^{2}$ Advanced Molecular Imaging, Eunice Kennedy Shriver National Institute of Child Health and Human \\ Development, NIH. \\ ${ }^{3}$ Microscopy \& Imaging Center (MIC), Eunice Kennedy Shriver National Institute of Child Health and \\ Human Development, NIH.
}

We report on how it is possible to flash-freeze, stain and image at very high magnifications microtubules stabilized with Taxol, using high electron doses, without recording modifications during illumination. This staining mechanism is used since a few years to consistently result in well-defined microtubules [1-2] for tomography experiments. Tentatively, the unexpected dose resistance after elimination of unbound water from the grids, by sublimation before irradiation, may reflect changes in absorption of the materials in the sample at wavelengths larger than visible light. Such radiation would result from parasitic scattering of electrons from the heavy metals in these samples. Diameter of resulting microtubules measured between 25 and $32 \mathrm{~nm}$. High electron dose is reflected in the high definition of these images, acquired with Poisson counting statistics. With such definition, some deformations of microtubule, possibly induced by interaction with stain or substrate, appear. As well, many irregularities on the surface of these microtubules are also present. Because of the high purity of this sample, the large constructs found on the surface of supporting film may derive from an unexpected association of tubulin, possibly involving material from the substrate. These large aggregates bind the stain. Very small-size (diameter $\sim 20 \AA$ ) globular stained aggregates were found in a control experiment using bovine-serumalbumin (BSA) instead of tubulin. BSA is known to spontaneously aggregate.

$11 \mu \mathrm{l}$ of solution of Taxol stabilized microtubules from pure $(>95 \%)$ rat brain tubulin was deposited using a calibrated pipette on a holey or C-only 200-mesh copper grid (EMS), while it was held by forceps for $\sim 1$ minute. Solution resulted from $8 \mu \mathrm{l}$ of microtubules mixed to $3 \mu \mathrm{l}$ of colloidal gold $(3 \mathrm{~nm})$ on grid. After wicking gently excess, $6 \mu \mathrm{l}$ of uranyl acetate $(1 \% \mathrm{~m} / \mathrm{v})$ was deposited for $\sim 30$ seconds on grid held by forceps. Then two $8 \mu \mathrm{l}$ drops of methyl-amine tungstate (NanoW, Nanoprobes), diluted 1:50, were used. The first drop was in contact with the grid $\sim 3 \mathrm{~s}$ while the second one $\sim 30 \mathrm{~s}$. As the second drop of diluted NanoW was deposited, the grid was quickly mounted in a Leica plunge freezer, blotted on the side, and rapidly $(<3 \mathrm{~s})$ immersed in liquid ethane. Samples were stored for 1-3 days in liquid nitrogen (LN) before use. For experiments, grids were inserted in a Gatan cryo-holder (Model 626) loaded with LN. After insertion in the Jeol 1400 TEM, temperature was raised $5^{\circ} \mathrm{C} / \mathrm{min}$ to $-102^{\circ} \mathrm{C}$ and then $1{ }^{\circ} \mathrm{C} / \mathrm{min}$ to $-92^{\circ} \mathrm{C}$. Sample was kept for $\sim 1.2$ hours at $-92^{\circ} \mathrm{C}$ before switching on electron beam at $120 \mathrm{keV}$. Vacuum was optimal when beam illuminated the grid. Imaging operations were complicated by the fact that sample was not flat because of membranaceous stained material. Therefore, the accessible angular range for tomography measurement was limited. Tomogram in Figure 3 was measured over $+55^{\circ}$ and $-52^{\circ}$ using a $1^{\circ}$ angular step and $5,000 \mathrm{e}^{-} / \mathrm{nm}^{2}$ per image. Total dose was $\sim 10^{6} \mathrm{e}^{-} / \mathrm{nm}^{2}$; beam was not blanked during motions. Defocus was set in SerialEM software to $0 \mu \mathrm{m}$. Resulting images were aligned and tomogram was reconstructed using the back-projection method (Etomo [3]).

Figures $1 \mathrm{~A}, \mathrm{~B}$ and $\mathrm{C}$ report microtubules prepared as described. Inside the tubular shapes an inner structure correspondent to protofilaments is seen. In Figure 1B and 1C the broken tubular structure reveals arrangement of constituent subunits in the inner part of the microtubules. Dimensions and shape of subunits (inset Figure 1B) are compatible with former results on microtubules structure. Unexpectedly, membranaceous or unstructured material not completely transparent to electrons is found, also covering or surrounding microtubules (Figure 3). Virtual 
sections $3.6 \AA$ thick from tomogram relative to blue box in Figure 1 are displayed in Figure 3. A cut through the center of this (straighter) microtubule (diameter $\sim 30 \mathrm{~nm}$ ) reveals stained material somewhat uniformly covering the outside surface of this microtubule, while it is less thick in the inner wall. Appearance of microtubules did not change during the sustained irradiation intrinsic to these experiments. Microtubules imaged in the conditions described appear completely different from microtubules freeze-dried and imaged at room temperature (Figure 2). Such procedure results in collapsed structures, as reported by Chretien [4].

Further experiments are necessary to clarify origin of membranes and deposits, possibly stain related [5].

\section{References:}

[1] A Fera. TS Reese, J. Zimmerberg, DL Sackett, ACS Nano - submitted.

[2] A Fera, et al, Microsc. \& Microanal. 18 (2012), p. 1.

[3] JR Kremer, DN Kisielowski, JR. Journal of Structural Biology 116 (1996), p. 71.

[4] RH Wade and D Chretien, Journal of Struct. Biology, 110 (1993), p. 1.

[5] We thank especially Dr. Dan Sackett, Dr. Bechara Kachar, Dr. Thomas S. Reese and Dr. Paul Blank for the many useful discussions, support and intellectual contributions to this work.
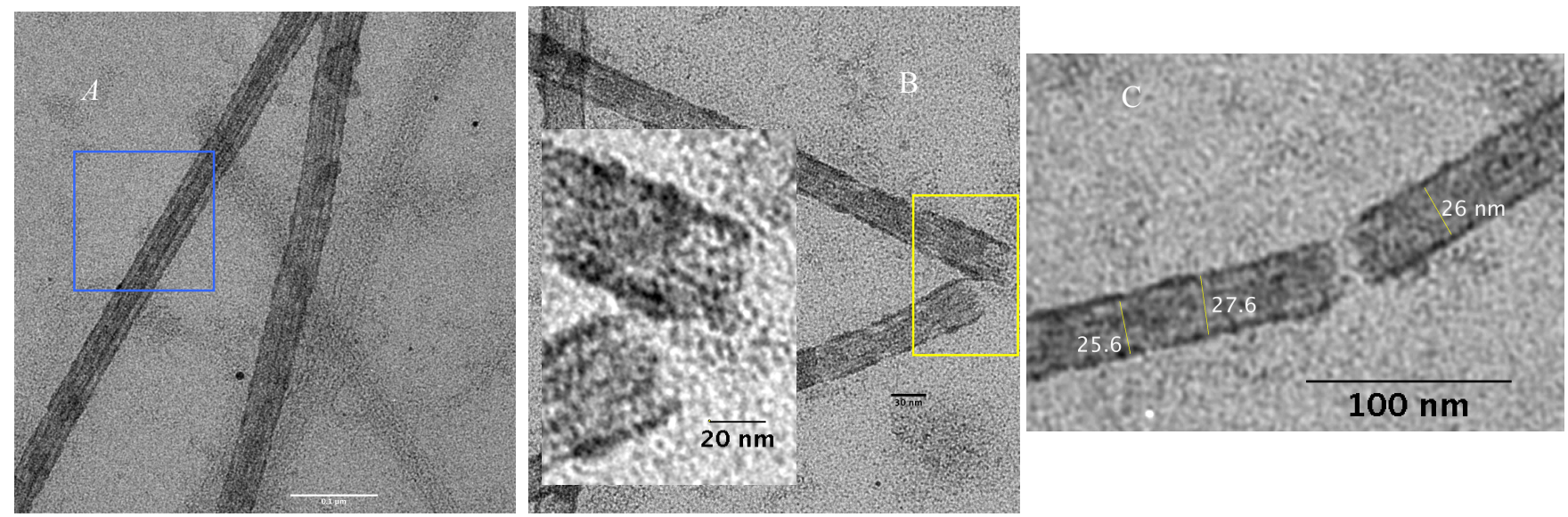

Figure 1. Microtubules stabilized with Taxol, stained and freeze-dried before imaging at $-92^{\circ} \mathrm{C}$. Diameter as expected, although stained (protein) material deposits on the surface. Virtual section from tomogram (below right) corresponds to blue frame in Figure 1A. Left inset in figure 1B corresponds to yellow frame. Apparent diameter of microtubules in pictures varies between $\sim 25.5$ and almost $32 \mathrm{~nm}$, especially near ruptured areas. Microtubules are well above substrate in all pictures.
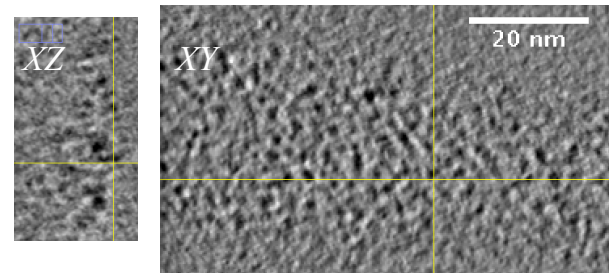

Figure 2. Tomogram extracts of Taxolstabilized microtubule, positive stained with UA and brought to room temperature overnight in electron microscope. The YZ lateral view on the left shows that microtubule is collapsed as in [4].

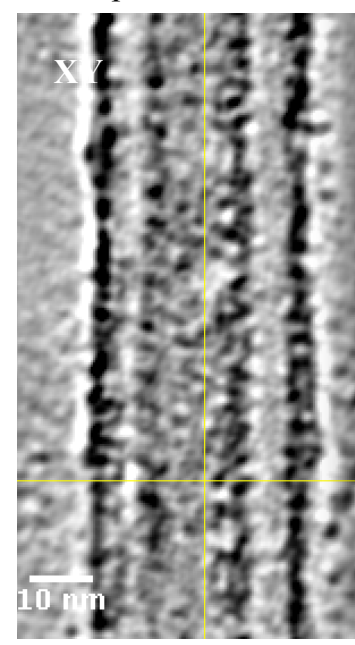

Figure 3. Virtual sections from tomogram. Microtubules freezedried for one hour inside microscope, without electron beam, imaged at $-92^{\circ} \mathrm{C}$ with a Jeol 1400 at $120 \mathrm{keV}$. The expected microtubule diameter and subunits dimensions are apparent in these calculated virtual sections $3.6 \AA$ thick. Angular range was limited (see text), so missing wedge prevents from studying the top and bottom protofilaments as in [1]. 